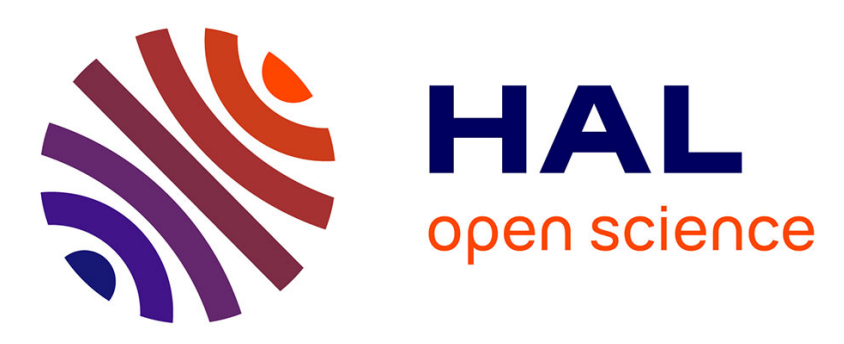

\title{
High intensity surface plasma waves, theory and PIC simulations
}

Michèle Raynaud, Anne Héron, Jean-Claude Adam

\section{To cite this version:}

Michèle Raynaud, Anne Héron, Jean-Claude Adam. High intensity surface plasma waves, theory and PIC simulations: https://doi.org/10.1088/1361-6587/aa8f13. Plasma Physics and Controlled Fusion, 2017, 60, pp.014021. 10.1088/1361-6587/aa8f13 . hal-03088515

\section{HAL Id: hal-03088515 https://hal.science/hal-03088515}

Submitted on 6 Jan 2022

HAL is a multi-disciplinary open access archive for the deposit and dissemination of scientific research documents, whether they are published or not. The documents may come from teaching and research institutions in France or abroad, or from public or private research centers.
L'archive ouverte pluridisciplinaire HAL, est destinée au dépôt et à la diffusion de documents scientifiques de niveau recherche, publiés ou non, émanant des établissements d'enseignement et de recherche français ou étrangers, des laboratoires publics ou privés. 
PAPER

High intensity surface plasma waves, theory and PIC simulations

To cite this article: M Raynaud et al 2018 Plasma Phys. Control. Fusion 60014021
Related content

- Tutorial

C Thaury and F Quéré

Short-pulse laser - plasma interactions P Gibbon and E Förster

Proton and neutron sources

J T Mendonça, J R Davies and M Eloy

View the article online for updates and enhancements. 


\title{
High intensity surface plasma waves, theory and PIC simulations
}

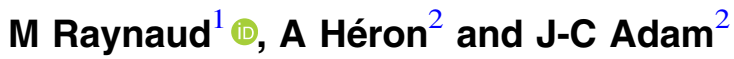 \\ ${ }^{1}$ Laboratoire des Solides Irradiés, CEA/DRF/IRAMIS, Ecole Polytechnique, CNRS, Université Paris- \\ Saclay, F-91128 Palaiseau, France \\ ${ }^{2}$ Centre de Physique Théorique, Ecole Polytechnique, CNRS, Université Paris-Saclay, F-91128 Palaiseau, \\ France
}

E-mail: michele.raynaud-brun@polytechnique.edu

Received 23 June 2017, revised 14 September 2017

Accepted for publication 25 September 2017

Published 31 October 2017

\begin{abstract}
With the development of intense $\left(>10^{19} \mathrm{~W} \mathrm{~cm}^{-2}\right)$ short pulses $(\leqslant 25 \mathrm{fs})$ laser with very high contrast, surface plasma wave (SPW) can be explored in the relativistic regime. As the SPW propagates with a phase velocity close to the speed of light it may results in a strong acceleration of electron bunches along the surface permitting them to reach relativistic energies. This may be important e.g. for applications in the field of plasma-based accelerators. We investigate in this work the excitation of SPWs on grating preformed over-dense plasmas for laser intensities ranging from $10^{19}$ up to $10^{21} \mathrm{~W} \mathrm{~cm}^{-2}$. We discuss the nature of the interaction with respect to the solid case in which surface plasmon can be resonantly excited with weak laser intensity. In particular, we show the importance of the pulse duration and focalization of the laser beam on the amplitude of the SPW.
\end{abstract}

Keywords: surface plasma wave, particle-in-cell simulations, fluid simualtions

(Some figures may appear in colour only in the online journal)

\section{Introduction}

Strong absorption of light connected with surface plasmon excitation of metallic diffraction grating is a well-known phenomenon in solids $[1,2]$. In plasma also, the possibility of electron surface waves [3] excitation by a laser beam on a structured target as an efficient means to improve the generation of fast particles has been investigated with success [4-12], in the laser intensity range of $10^{18-19} \mathrm{~W} \mathrm{~cm}^{-2}$. In plasma at solid density, the matching conditions for the first order excitation of surface plasma wave (SPW) on grating targets are obtained by analogy with the solid case. It is supposed to satisfy the Rayleigh condition $k=k_{0}\left(\sin \theta+\lambda_{0} / d\right)$ together with the dispersion relation [3] of the SPW in the cold plasma non-relativistic limit: $(k c / \omega)^{2}=\left(1-n_{\mathrm{e}} / n_{\mathrm{c}}\right) /\left(2-n_{\mathrm{e}} / n_{\mathrm{c}}\right)$ and $\omega=\omega_{0}$, where $k$ and $\omega$ are the wave vector and the frequency of the surface wave respectively, $k_{0}$ and $\omega_{0}$ are the wave vector and angular frequency of the laser field, $n_{\mathrm{e}}$ is the electron density and $n_{\mathrm{c}}=m$ $\omega_{0}^{2} / 4 \pi e^{2}$ the critical density, $d$ denotes the period of the grating surface density, $\theta$ is the incidence angle and $\lambda_{0}$ the wavelength of the laser. With the development of intense $\left(>10^{19} \mathrm{~W} \mathrm{~cm}^{-2}\right)$ short pulse $(\leqslant 25 \mathrm{fs})$ lasers with very high contrast [13], SPW which need to develop themselves a steep density gradient, can be explored in the highly relativistic regime. For short enough laser pulses and if the surface wave is robust, we can expect bunches of electrons to be accelerated to velocities that are almost equal to the phase velocity of the SPW that is close to the light velocity. This might lead to a pulse train of collimated very high quasi-mono-energetic electrons with possible applications in tabletop electron accelerators and ultrafast-electron spectroscopies $[14,15]$ which makes this approach very attractive. To achieve this goal, a full understanding of the surface wave excitation mechanism in the relativistic domain is needed.

Even for moderate intensities, there is no analytical dispersion relation for the SPW for gratings with a finite modulation depth. There exist at most few results obtained by perturbation theory. Most of the results come from numerical integration of Maxwell's equations with adequate boundary conditions $[1,2]$. No results at all are available in the relativistic regime where the situation is complicated by the presence of the $\mathbf{J} \times \mathbf{B}$ term in the equations. Nevertheless, numerical particlein-cell (PIC) simulations have provided evidence of SPW and related effects in the short pulse relativistic regime up to $10^{19} \mathrm{~W} \mathrm{~cm}^{-2}$ even if relativistic effects are not taken into 
account to determine precisely the matching conditions for the SPW excitation. In this work, we investigate the laser excitation of SPW on grating targets in the relativistic regime for laser intensities ranging from $10^{19}$ up to $10^{21} \mathrm{~W} \mathrm{~cm}^{-2}$ by means of PIC simulations. We discuss the nature of the interaction with respect to the solid case at moderate intensity. Large-scale two-dimensional PIC simulations, with realistic parameters compared to the experimental conditions, are first analyzed in the second section of this paper. Special attention is devoted to the spectral analysis of the fields and the nature of the surface modes is discussed. These results are next compared with those resulting from a relativistic fluid description in the third section. With this model, the role of the pulse temporal shape of the incident laser beam and of the grating geometry of the target is investigated. This work shows the influence of relativistic effects and of both the spatial and temporal shape of the beam on the surface plasma modes. It opens discussion about the exploitation of plasmonic effects in the high intense fields.

\section{PIC simulations}

In the following, we describe the results of 2D PIC simulations performed with the relativistic collisional code EMI2D [16]. The calculations were carried out for a slab of an overdense plasma with a thickness of $10 \mu \mathrm{m}$, an electron density $n_{\mathrm{e}}=120 n_{\mathrm{c}}$ and a steep density gradient along the perpendicular direction of the simulation plane. The plasma is initially described by a Maxwellian distribution with an electron temperature $T_{\mathrm{e}}=50 \mathrm{eV}$ and an ion temperature $T_{\mathrm{i}}=T_{\mathrm{e}} / 5$. The simulation parameters are chosen to be close to those of recent experiments [12]. The plasma target is assumed to be a Mylar foil modeled with an averaged $Z / A$ equals to $4 / 6$. In order to fulfill the matching condition of SPW excitation we have considered a modulated sinusoidal surface with a modulation periodicity $d\left(d=8.44 k_{0}{ }^{-1}\right)$ chosen such that the SPW will be excited at the incidence angle of $15^{\circ}$. The dip-totip modulation depth $h$ of the modulated surfaces is $h=2.094 k_{0}{ }^{-1}$ that is $h / d=0.25$. The incoming oblique laser beam is P-polarized as this is the only polarization that gives rise to the excitation of SPW on gratings. The laser beam is focused on the center of the plasma surface $\left(x k_{0}=\right.$ $y k_{0}=0$ ) with a focal spot of $30 k_{0}^{-1}$ and a pulse duration of about $25 \mathrm{fs}$. Several values of the laser intensity were considered hereafter ranging from $2.6 \times 10^{19} \mathrm{~W} \mathrm{~cm}^{-2}\left(a_{0}=4.4\right)$ up to $1.3 \times 10^{21} \mathrm{~W} \mathrm{~cm}^{-2}\left(a_{0}=31\right)$. The simulation box considered is large: $340 \lambda_{0}$ along the $y$-direction $\left(2136 k_{0}{ }^{-1}\right)$ and $103 \lambda_{0}$ along the $x$-direction both at the front and the rear ends of the plasma sheet (resulting in $1330 k_{0}{ }^{-1}$ for the dimension of the whole box in the $x$-direction). We choose a grid size of $k_{0} \Delta x=k_{0} \Delta y=0.018 \approx 20 k_{0} \lambda_{D}$ for which the code EMI2D remains stable and the number of particles per cell was 120. In the results produced hereafter the plasma surface is located at $k_{0} x=0$ and the maximum impulsion of the incident laser is impinging on the plasma surface at location $\left(k_{0} x=0, k_{0} y=0\right)$ and at the time $\omega_{0} t=0$. In all the figures, the units are $k_{0}{ }^{-1}$ for space and $\omega_{0}^{-1}$ for time.

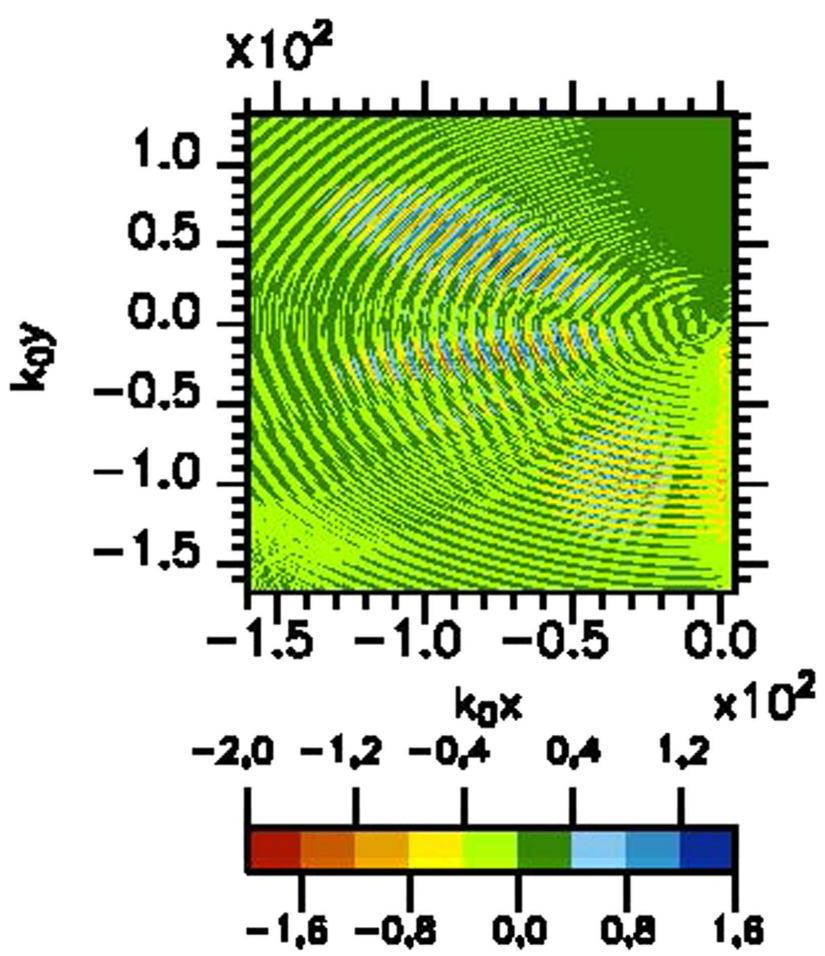

Figure 1. PIC simulation for $a_{0}=4.4$ in the $\left(x k_{0}, y k_{0}\right)$ space in front of the plasma surface located at $x k_{0}=0, B_{z}$ component of the magnetic field (at time $\omega_{0} t=110$ ).

The time dependent surface wave excitation has been analyzed for $a_{0}=4.4,13$ and 31 . We first note that even if the grating is smoothed when $a_{0}$ increases, its periodicity is maintained during the first cycles of the laser plasma interaction. Consequently, the SPW excitation still occurs in this range of high laser intensity. We also observe that the maximum amplitude of the SPW grows more slowly than the laser amplitude. The analysis of the magnetic field $B_{z}$ shows that, as expected due to the grating parameters, at times greater than the time at which the laser impacts the surface, several orders of reflection (from zero, to third order) are observed in the figure 1. Their positions depend on the laser incidence in agreement with Rayleigh formula. Near the plasma surface there is also an extra field structure located on the plasma surface as can be seen in the figure 2(a) in case $a_{0}=4.4$ and for $\omega_{0} t=0$. The spectrum in $k$ space of this structure is centered on $k_{y} / k_{0} \approx 1.01$ and has a width of the same order as those of the incident mode as seen in the figures 2(b) and (c). This structure around $k_{y} / k_{0} \approx 1.01$ is highly localized close to the plasma surface. It can be attributed to the SPW for which we expect $k_{y}=1.004 k_{0}$ considering the dispersion relation [3] of the SPW in the cold plasma non-relativistic limit. Note that the dispersion relation supposes an incident monochromatic wave. Finally, a puzzling question concerns the local amplitude of the SPW. We observe no significant amplification with respect to the incoming light and the SPW amplitude is much smaller than the one that can be expected in solid-state physics.

However, the previous PIC 2D simulations with a short pulse Gaussian temporal shape ( $25 \mathrm{fs})$ and spatial focused Gaussian transverse profile $\left(30 k_{0}{ }^{-1}\right)$ were performed in 

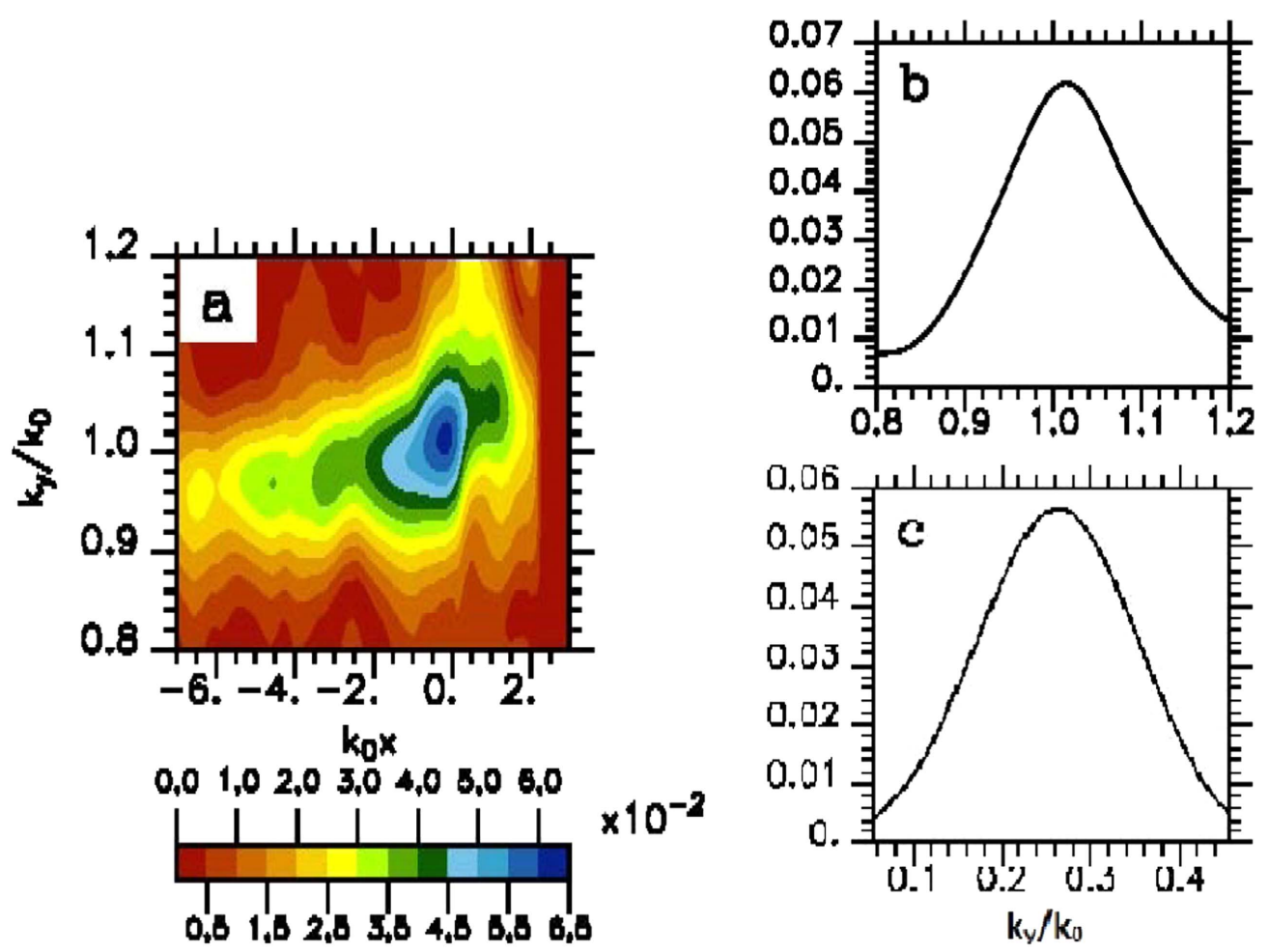

Figure 2. PIC simulation for $a_{0}=4.4$ : (a) $B_{z}$ component of the magnetic field (at time $\omega_{0} t=0$ ) in the Fourier space $\left(x k_{0}, k_{y} / k_{0}\right)$, (b) maximum amplitude of the module of the field in the Fourier space as a function of $k_{y} / k_{0}$ on the vacuum side for $x k_{0}=-0.2$, (c) maximum amplitude of the incoming field in the Fourier space as a function of $k_{y} / k_{0}$ before impinging the plasma surface.

conditions very far from the theoretical conditions used in solid state physics. This induces a distribution of modes in the Fourier space that complicated the analysis. We thus complement our investigation in the following using a relativistic 2D fluid description which is well adapted to make comparison with the solid-state case at moderate intensity.

\section{Fluid description}

The fluid model of plasma that we use here is strictly identical to the Drude model of solid-state physics. Consequently, it yields the same theoretical dispersion for SPWs. We consider that the grating is made of plasma of electrons neutralized by a fixed background of ions. The presence of a collisional dissipation is described by a collisional term $\nu_{\mathrm{ei}}$.

In the non-relativistic case, the equation of momentum conservations multiplied by the plasma density $n_{\mathrm{e}}$ gives the evolution equation for current used as a source term in Maxwell equation. This equation is written:

$$
\frac{\partial j_{\mathrm{e}}}{\partial t}=-\frac{n_{\mathrm{e}} e}{m_{\mathrm{e}}} E-\nu_{\mathrm{ei}} j_{\mathrm{e}} \quad \text { with } \quad j_{\mathrm{e}}=n_{\mathrm{e}} e v_{\mathrm{e}} .
$$

In the relativistic case, the Lorentz force and the relativistic factor $\gamma$ must be added. The equation for the current becomes:

$$
\frac{\partial j_{\mathrm{e}}}{\partial t}=-\frac{n_{\mathrm{e}} e}{m_{\mathrm{e}} \gamma}\left(E+v_{\mathrm{e}} \times B\right)-\nu_{\mathrm{ei}} j_{\mathrm{e}} .
$$

The code used to integrate Maxwell equations is the same one as the one used in EMI2D, the only difference being the way the current is obtained. The identity between the present model and the ones based on the Drude model in solid-state physics implies that all the known results of the grating theory are reproducible in this framework.

One of the most remarkable results widely studied in the literature is the so-called Wood anomaly (1902) which corresponds to the total absorption of the incident electromagnetic wave associated to the excitation of a surface wave for extremely specific conditions of interaction of the incident electromagnetic wave with the grating [2]. The present model reproduces accurately the results published in the [2] for the same conditions of interaction i.e. a perfectly monochromatic source of indefinite duration in order to obtain a stationary state. However in the present problem of interest, as already indicated, the source is neither monochromatic (the pulse is finite both in the transverse direction and in time) nor stationary. This makes the standard results inapplicable and justifies the present approach.

We consider a set of parameters similar to those of the PIC simulations $\left(a_{0}=4.4, h / d=0.25\right)$. First, in order to simplify the analysis, we consider an incident plane wave with infinite pulse duration to reach a stationary regime of interaction as in solid surface plasmon excitation [1, 2]. Boundary conditions are periodic in the $y$ direction. In order to obtain accurate results care has to be taken to insure that the size of the system in the $y$ direction is a multiple of both the wavelength of the laser and that of the SPW. This conducts to 

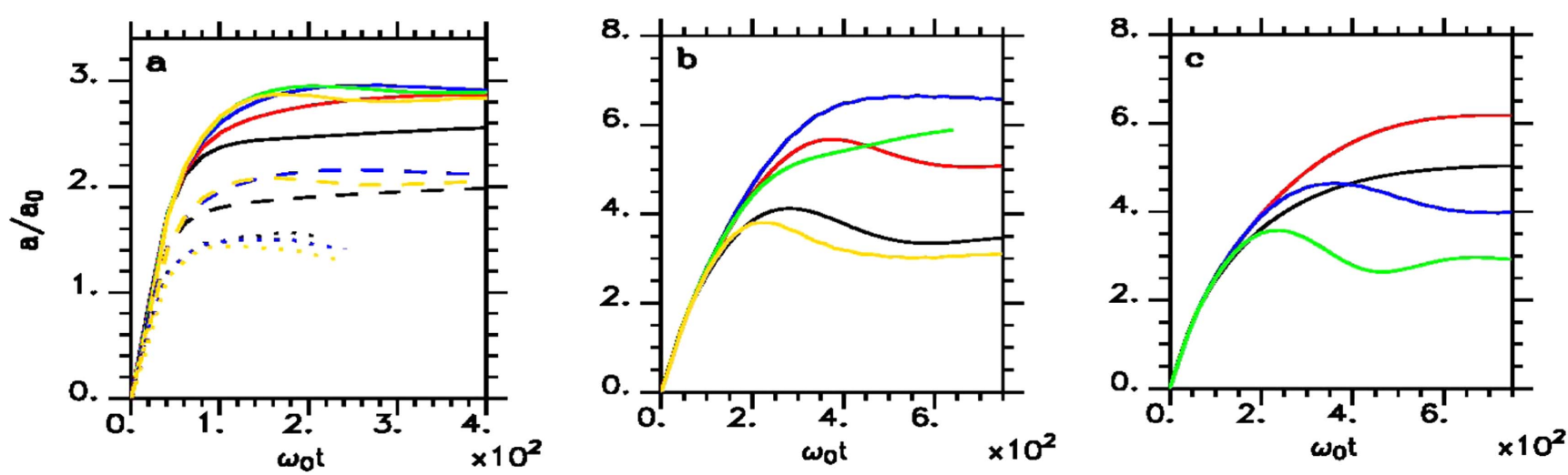

Figure 3. Maximum amplitude evolution with the time of the module of the $B_{z}$ field (normalized to $a_{0}$ ) in the Fourier space of the modes $k_{y} / k_{0}=1.004$ (black), 1.009 (red), 1.015 (blue), 1.02 (green) and 1.26 (yellow). (a) $a_{0}=4.4, h / d=0.25$, full line: fluid simulations with $\nu_{\mathrm{ei}} / \omega_{0}=0.4$, dashed line: fluid simulations with $\nu_{\mathrm{ei}} / \omega_{0}=1.8$, dotted line: PIC simulations, (b) fluid simulations with $a_{0}=4.4, \nu_{\mathrm{ei}} / \omega_{0}=0.4$ and $h / d=0.1$, (c) fluid simulations with $a_{0}=0.1, \nu_{\mathrm{ei}} / \omega_{0}=0.4$ and $h / d=0.1$
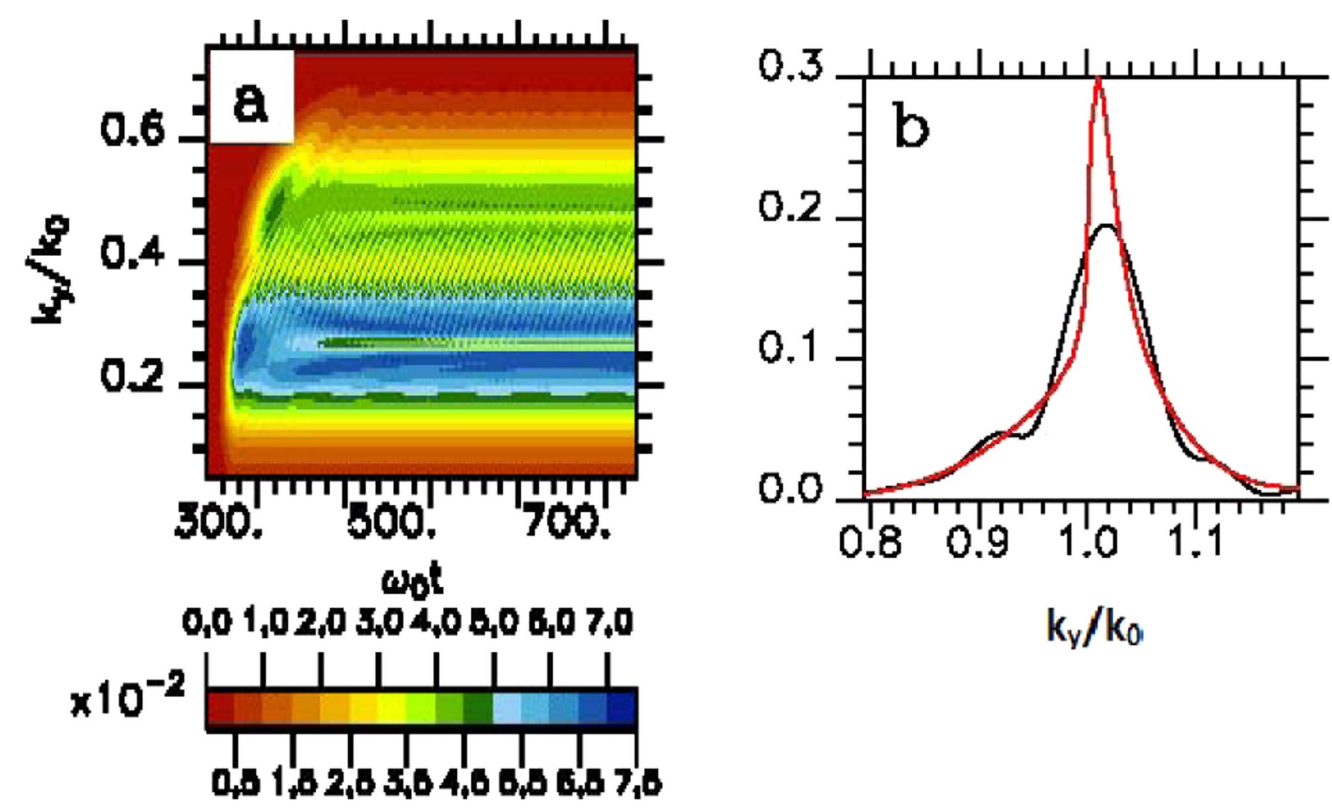

Figure 4. Fluid simulation $\left(a_{0}=4.4, h / d=0.1, \nu_{\mathrm{ei}} / \omega_{0}=1.8\right.$ ): (a) Fourier space of reflected field as a function of time, (b) maximum amplitude of the module of the field in the Fourier space as a function of $k_{y} / k_{0}$ for $\omega_{0} t=120$ and 700 .

large systems in $y$. In this case, the time $\left(\omega_{0} t=0\right)$ corresponds to the time where the pulse is impinging the plasma surface $\left(k_{0} x=0\right)$. Simulations using both the PIC code and the fluid model were performed in this regime. The maximum amplitude evolution with the time of the module of the $B_{z}$ field normalized to the incident field amplitude in the Fourier space is plotted in the figure 3(a) for five incident angles ranging from $15^{\circ}$ up to $16.4^{\circ}$ of the laser field corresponding to $k_{y} / k_{0}$ of the SPW ranging from 1.004 up to 1.26 . The plots in continuous line correspond to the fluid model where $\nu_{\mathrm{ei}} / \omega_{0}=0.4$, those in dashed line to $\nu_{\mathrm{ei}} / \omega_{0}=1.8$ and those in dotted line to PIC code results. We observe that increasing $\nu_{\text {ei }} / \omega_{0}$ tends to decrease the amplitude reached in the saturation regime. This outlines the arbitrary choice for the value of $\nu_{\mathrm{ei}} / \omega_{0}$ in the fluid model. Contrastingly, in the PIC simulations the collisions are characteristic of the plasma.
Their suppression does not change the results due to the fast heating of the electrons in the skin depth. At last, we also note that the surface wave excitation is broad in $k$-space in the two descriptions for the set of parameters considered here.

In solids, it is well known that the $h / d$ parameter plays a major role in the surface plasmon excitation [1]. Thus we next compare the previous case for which $h / d=0.25$ with the one with $h / d=0.1$ with the grating periodicity unchanged. The results obtained are shown in the figure $3(\mathrm{~b})$. We observe an increase in the maximum value of the mode amplitude by a factor 2 in the best case $\left(k_{y} / k_{0}=1.015\right)$. The $k_{y} / k_{0}$ dependence becomes also narrower when $h / d=0.1$. Moreover the mode for which $k_{y} / k_{0}=1.004$ (which satisfy the SPW conditions of excitation for a cold non-relativistic plasma) have an amplitude smaller by a factor 1.5 than the mode $k_{y} / k_{0}=1.015$. It can be due to relativistic detuning which is 


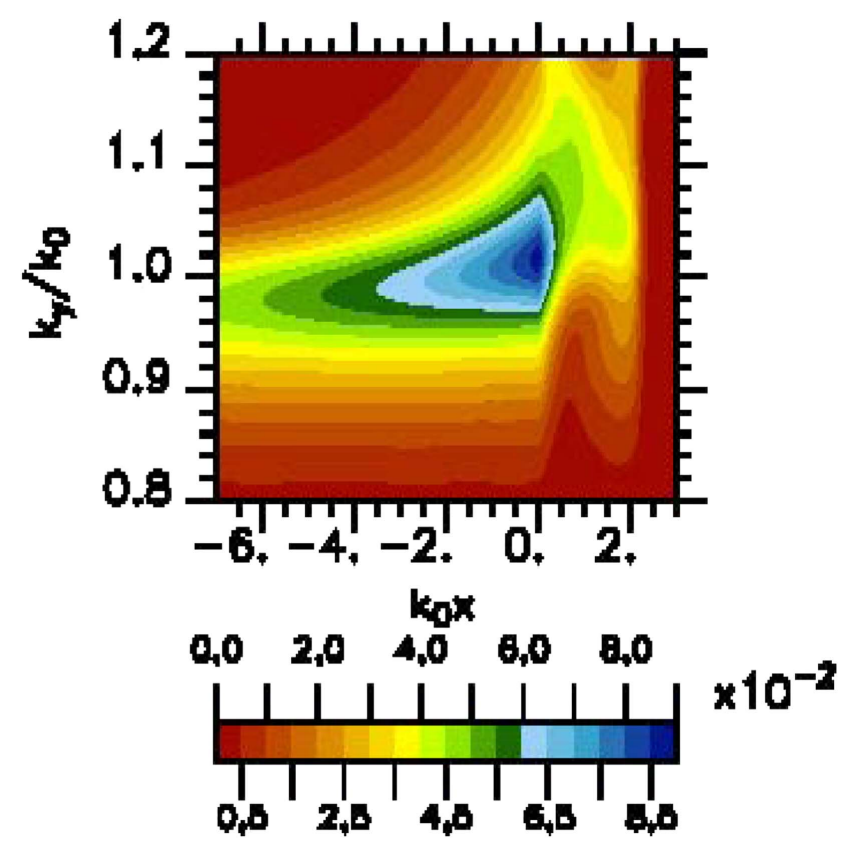

Figure 5. Fluid simulation with the same parameters as in figure 1(a) (at $\omega_{0} t=0$ ): $B_{z}$ component of the magnetic field in the Fourier space $\left(x k_{0}, k_{y} / k_{0}\right)$.

already expected at this laser intensity and shifts the value of the wave vector towards a higher value. This shift can be estimated by replacing $\omega_{p}$ by $\omega_{p} / \gamma$ where $\gamma$ is the Lorentz factor. One obtains the modified value $k_{y} / k_{0}=1.014$ for $a_{0}=4.4$. This is in good agreement with our result. Decreasing next $a_{0}$ to 0.1 , we observe a quasi linear dependence of the maximum mode amplitude with $a_{0}$. Note however that the mode for which $k_{y} / k_{0}=1.015$ is no longer the one having the highest amplitude as seen in the figure 3(c) the highest one being in this case the mode $k_{y} / k_{0}=1.009$. This shift clearly demonstrates the role of the relativistic effects. Note here that the mode $k_{y} / k_{0}=1.004$ that satisfies the conditions for SPW excitation for a plane surface of interaction has a smaller amplitude. It can be induced by the effect of the finite depth of the grating that is not taken into account in the SPW dispersion relation calculation derived in [3].

Finally, we observed that a long time is needed to reach the stationary regime. For short pulse duration of the order of that used in experiments, $\sim 25 \mathrm{fs}$, the maximum amplitude of the mode $\left(k_{y} / k_{0}=1.015\right)$ approximatively equals $2 a_{0}$ which is three time smaller than the value in the stationary regime.

At last, we perform fluid simulations with an infinite incident beam focused on the plasma surface with the same focal spot as in the PIC simulations. The results are shown in figure 4. In figure 4(a), we observe a significant depletion of the spectrum of the reflected wave around $k_{y} / k_{0}=0.26$. It can be correlated with the appearance of the surface mode around 1.009 (see figure 4(b)). This depletion is very narrow in $k_{y}$, only a small fraction of incident energy is transferred to SPW. During the laser plasma interaction, the width of the modes distribution around $k_{y} / k_{0} \sim 1$ decreases as seen on the figure 4(b).

Finally, we consider the same laser field as in the second section, with a duration pulse of $25 \mathrm{fs}$ and a focal spot of $30 k_{0}{ }^{-1}$. Using the parameters corresponding to these of the PIC simulation, we find as shown in the figure 5 that the shape of the field is quite similar to the one obtained in the PIC simulation in figure 2(a). Note that the amplitude depends on the chosen $\nu_{\mathrm{ei}} / \omega_{0}$.

\section{Conclusion}

In this work, we have investigated the laser excitation of SPW on grating preformed over-dense plasma in the relativistic regime ranging from $10^{19}$ up to $10^{21} \mathrm{~W} \mathrm{~cm}^{-2}$. We have compared collisional 2D PIC simulations with fluid description and have shown that the fluid description is useful to optimize the conditions of interaction with gratings. We have evidenced the role of the relativistic detuning that shifts the SPW vector towards a higher value. Nevertheless the SPW seems to be robust up to $a_{0}=31$. We have also shown that as in solid the ratio of the modulation depth, $h$, to the periodicity, $d$, is a crucial parameter. Decreasing $h / d$ from 0.25 to 0.1 increases the SPW field amplitude, so that this parameter can be used to optimize the SPW excitation. Finally, we have pointed out that although the use of short pulse durations is needed to preserve the grating shape during the interaction in order to excite the SPW, the short pulse limits the SPW amplitude. Also, using a focused pulse in the experiments induces a spectral width larger than that of the SPW. Consequently, the energy of the SPW is reduced.

\section{Acknowledgements}

All the PIC simulations were performed using the HPC BlueGene/Q IDRIS resources from the GENCI Grant number 2015-2017/056851 and the fluid simulations were performed on local clusters of the CPHT laboratory.

\section{ORCID iDs}

M Raynaud (ib https://orcid.org/0000-0002-0489-1002

\section{References}

[1] Hutley M C and Maystre D 1976 Opt. Commun. 1943

[2] Popov E and Tsonev L 1990 Surf. Sci. 230290

[3] Kaw P K and McBride J B 1970 Phys. Fluids 131784

[4] Willingale L et al 2011 Phys. Rev. Lett. 106105002

[5] Naseri N, Pesme D and Rozmus W 2013 Phys. Plasmas 20 103121

[6] Willingale L et al 2013 New J. Phys. 15025023

[7] Tian Y et al 2012 Phys. Rev. Lett. 109115002 
[8] Bigongiari A, Raynaud M, Riconda C and Héron A 2013 Phys. Plasmas 20052701

[9] Ceccotti T et al 2013 Phys. Rev. Lett. 111185001

[10] Wang W Q, Yin Y, Yu T P, Xu H, Zhuo H B, Ma Y Y and Shao F Q 2014 Phys. Plasmas 21123112

[11] Riconda C, Raynaud M, Vialis T and Grech M 2015 Phys. Plasmas 22073103
[12] Fedeli L et al 2016 Phys. Rev. Lett. 116015001

[13] Dromey B, Kar S, Zepf M and Foster P 2004 Rev. Sci. Instrum. 75645

[14] Tokita S, Hashida M, Inoue S, Nishoji T, Otani K and Sakabe S 2010 Phys. Rev. Lett. 1052015004

[15] Liu J S et al 2011 Phys. Rev. Lett. 107035001

[16] Héron A and Adam J-C 2015 Phys. Plasmas 22072306 